\title{
Behavioral Factors Mediating the Impact of Educational Attainment on Incident Heart Failure
}

\author{
- A Mediation Analysis -
}

\author{
Yifen Lin, PhD; Shaozhao Zhang, MD; Shuyi Wang, MD; Xiangbin Zhong, PhD; \\ Yuqi Li, PhD; Zhenyu Xiong, PhD; Xiuting Sun, PhD; Yiquan Huang, MD; \\ Yongqiang Fan, MD; Yue Guo, PhD; Huimin Zhou, MD; Daya Yang, PhD; \\ Menghui Liu, MD; Xingfeng Xu, MD; Xiaodong Zhuang, MD, PhD; Xinxue Liao, MD, PhD
}

\begin{abstract}
Background: To examine the association of low educational attainment with incident heart failure (HF) and explore potential behavioral mediators of the causal pathway.
\end{abstract}

\begin{abstract}
Methods and Results: A total of 12,109 participants in the Atherosclerosis Risk in Communities Study (ARIC) were included. Educational attainment was measured at baseline, and the risk of HF across educational attainment groups was assessed by Cox proportional hazards models. Using mediation analysis, we evaluated the mediating role of behavioral factors in the causal pathway between educational attainment and HF. During a median follow-up of 25.1 years, 2,407 cases (19.9\%) of HF occurred. Educational attainment showed an inverse association with HF risk (hazard ratio $(\mathrm{HR}), 1.41$; 95\% confidence interval $(\mathrm{Cl}), 1,26-1.57$ for low educational attainment; $\mathrm{HR}, 1.13 ; 95 \% \mathrm{Cl}, 1.02-1.25$ for medium educational attainment). In the mediation analysis, the association between educational attainment and HF was partially mediated by income, waist-to-hip ratio, current smoking, body mass index, current drinking, sports and physical activity, which explained $24.3 \%, 20.2 \%, 13.8 \%, 10.1 \%, 7.7 \%, 7.3 \%$ and $4.5 \%$, respectively, of the relationship. In total, all mediators contributed $56.3 \%$ of the total effect.
\end{abstract}

Conclusions: Low educational attainment was associated with increased risk for HF. Income, obesity and current smoking mediated a great proportion of the total effect of educational attainment on HF. Our results provide underlying insights for the development of targeted public health interventions to reduce educational disparities on HF incidence.

Key Words: Educational attainment; Heart failure; Income; Mediation analysis; Obesity

$\mathbf{H}$ eart failure (HF) is one of the most common cardiovascular diseases (CVD) and remains the leading cause of death worldwide. ${ }^{1}$ Although the treatment and prognosis of HF have been improving for decades, there are still high burdens of increasing morbidity, mortality and medical expenditure of HF. ${ }^{2}$ Recent studies indicate lower socioeconomic status, such as lower educational attainment, is associated with a higher risk of HF. ${ }^{3,4}$ Educational attainment, an easily measurable marker of socioeconomic status, is usually completed in early life, but has a long-term impact on the life course. $.5,6$ However, few studies have focused on the potential mech-

\section{Editorial p1553}

anism underlying the increased risk of lower educational attainment on HF. Therefore, the potential causal pathway needs to be clarified, which will enable us to identify the mediating risk factors and develop specific public health strategies for eliminating these risk factors. ${ }^{7.8}$

Previous studies show that the risk of CVD attributed to educational attainment is partially mediated by behavioral factors (i.e., smoking, drinking, sports, body mass index (BMI), waist-to-hip ratio (WHR) and income). ${ }^{9-11}$ However,

Received February 16, 2021; revised manuscript received April 12, 2021; accepted April 19, 2021; J-STAGE Advance Publication released online June 15, 2021 Time for primary review: 13 days

Cardiology Department (Y. Lin, S.Z., X. Zhong, Y. Li, Z.X., X.S., Y.H., Y.F., Y.G., H.Z., D.Y., M.L., X.X., X. Zhuang, X.L.), Department of Rheumatology (S.W.), First Affiliated Hospital of Sun Yat-Sen University, Guangzhou; NHC Key Laboratory of Assisted Circulation, Sun Yat-Sen University, Guangzhou (Y. Lin., S.Z., X. Zhong., Y. Li, Z.X., X.S., Y.H., Y.F., Y.G., H.Z., D.Y., M.L., X.X., X. Zhuang, X.L.), China

The first two authors contributed equally to this work (Y. Lin, S.Z.).

Mailing address: Xinxue Liao, MD, PhD, and Xiaodong Zhuang, MD, PhD, Cardiology Department, First Affiliated Hospital of Sun Yat-Sen University, 58 Zhongshan 2nd Road, Guangzhou 510080, China. E-mail: liaoxinx@mail.sysu.edu.cn (X.L.); zhuangxd3@mail.sysu.edu.cn (X. Zhuang)

All rights are reserved to the Japanese Circulation Society. For permissions, please e-mail: cj@j-circ.or.jp

ISSN-1346-9843 


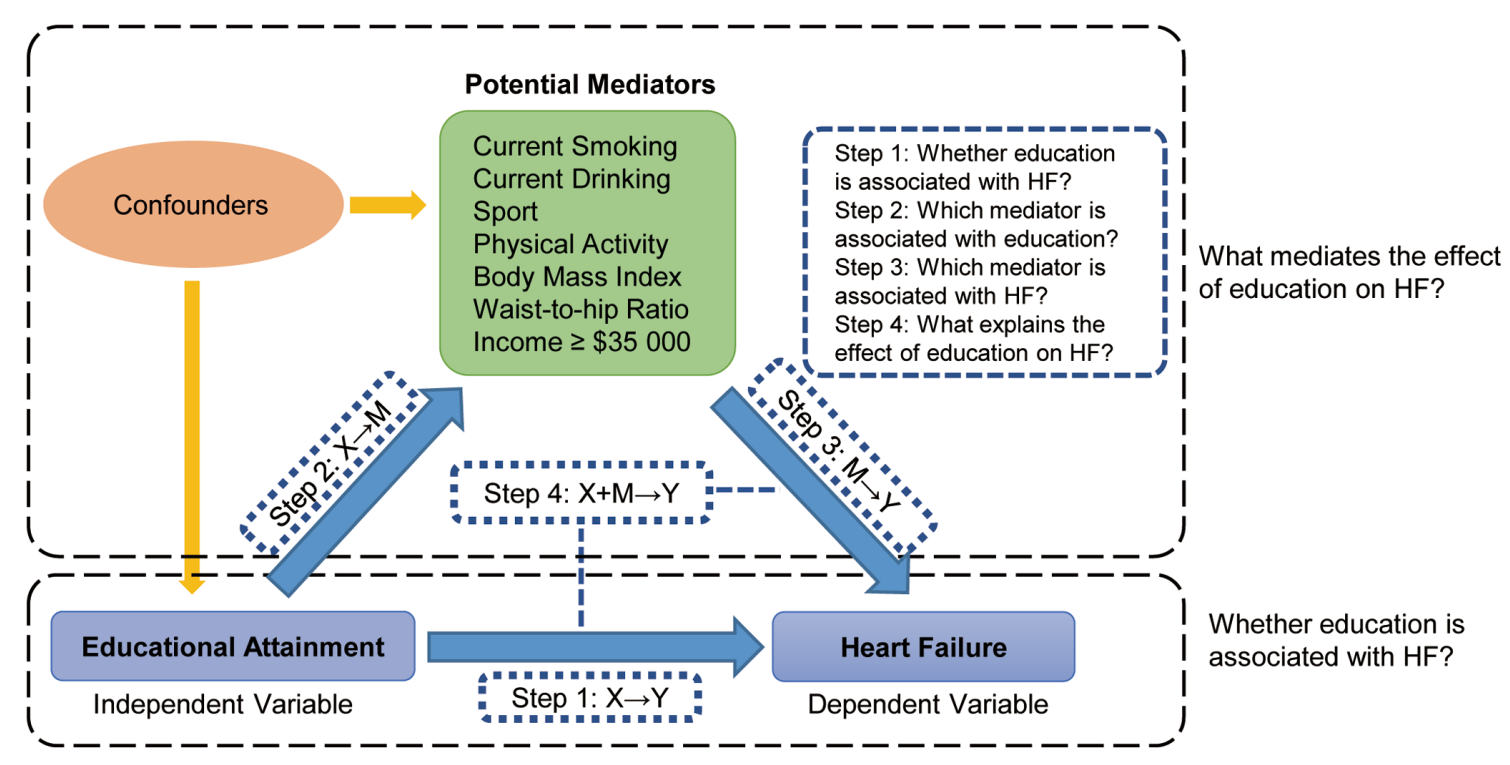

Figure 1. Potential mediating pathways between educational attainment and heart failure, divided into direct and indirect pathways. The mediation analysis comprised 4 steps: step 1, explore the association between educational attainment and heart failure; steps $2-4$, identify the mediators of the indirect pathways and quantify their contribution.

it is still unclear whether the association between educational attainment and $\mathrm{HF}$ is mediated by these risk factors, and, if so, to what extent does each risk factor mediate. In the present study, we used causal mediation analysis to examine whether the relationship is mediated by selected behavior factors and we also assessed the proportion mediated. We explored the association between educational attainment and risk of incident HF using data from the ARIC (Atherosclerosis Risk in Communities) study. We further identified the modifiable behavioral mediators involved in the association and assessed their contributions.

\section{Methods}

\section{Study Population}

The ARIC is a prospective investigation designed to identify the risk factors of CVD in 15,792 men and women.12 The datasets of ARIC were obtained at https://sites.cscc. unc.edu/aric/ according to the application procedure. The participants, aged 45-65 years, were initially recruited from 4 US communities (Forsyth County, NC; Jackson, MS; suburbs of Minneapolis, MN; and Washington County, MD) in 1987-1989 for a baseline visit. The followup visits were conducted during 1990-1992 (visit 2), 19931995 (visit 3), 1996-1998 (visit 4), and 2011-2013 (visit 5). The each institutional review board (IRB) approved the ARIC protocol, and all participants gave informed consent at each visit.

For this analysis, all participants at baseline were evaluated and we excluded those who were other than white or black race $(n=103)$, those with prevalent $\mathrm{HF}$ or missing data of HF $(n=1,273)$, those with missing educational information $(n=20)$, those with missing data of the mediated variable $(n=1,508)$ and other covariates $(n=779)$. Overall, we finally included 12,109 participants in our analysis.

\section{Educational Attainment Assessment}

Educational attainment information was obtained through questionnaire during the baseline visit. Educational attainment was categorized into 3 levels: low (0 years education, basic education or high school without completion), medium (high-school degree or vocational school) and high (attending or completed college, graduate or professional school).

\section{Outcome}

Incident HF was defined as the first occurrence of hospitalized HF according to International Statistical Classification of Diseases codes in any position (ICD-9 code 428) or a death certificate with death from HF in any position (ICD-9 and ICD-10, codes 428 and 150). Time interval from baseline to incident event was calculated and recorded as the follow-up time.

\section{Potential Mediators}

We chose some behavioral factors previously reported to be associated with HF and that could be susceptible to educational attainment as potential mediators in our model: income, smoking, drinking, sports, physical activity, BMI and WHR. ${ }^{13-16}$ All potential mediators were assessed at visit 1 . Information on smoking, drinking and income were self-reported. Income per year was categorized into 2 groups, $<$ US $\$ 35,000$ and $\geq U S \$ 35,000$. The validated Baecke questionnaire were used to assess sports and physical activity. ${ }^{17,18}$ The sports score was calculated based on the time and frequency of overall sporting activity and frequency of sweating with exercise, reflecting the intensity and frequency of exercise during leisure time. Physical activities were evaluated as frequency of watching television, walking, biking and time spent on bike or walking to work or shops. Sports score and physical activities score, as continuous variables, ranged from 1 to 5 and 1 to 4.5 , respectively. Height, weight, waist and hip circumference were 


\begin{tabular}{|c|c|c|c|c|c|}
\hline \multirow[b]{2}{*}{ Characteristic } & \multirow{2}{*}{$\begin{array}{c}\text { Total } \\
(n=12,109)\end{array}$} & \multicolumn{3}{|c|}{ Educational attainment } & \multirow[b]{2}{*}{$P$ value } \\
\hline & & $\begin{array}{c}\text { Low } \\
(n=2,664)\end{array}$ & $\begin{array}{c}\text { Medium } \\
(n=5,007)\end{array}$ & $\begin{array}{c}\text { High } \\
(n=4,438)\end{array}$ & \\
\hline Age, years & $54.2 \pm 5.8$ & $56.0 \pm 5.6$ & $54.0 \pm 5.6$ & $53.4 \pm 5.7$ & $<0.001$ \\
\hline Male & $5,621(46.4)$ & $1,282(48.1)$ & $2,037(40.7)$ & 2,302 (51.9) & $<0.001$ \\
\hline White & $9,386(77.5)$ & $1,538(57.7)$ & $4,241(84.7)$ & $3,607(81.3)$ & $<0.001$ \\
\hline $\mathrm{BMI}, \mathrm{kg} / \mathrm{m}^{2}$ & $27.4 \pm 5.2$ & $28.8 \pm 6.0$ & $27.3 \pm 5.1$ & $26.9 \pm 4.1$ & $<0.001$ \\
\hline WHR & $0.925 \pm 0.078$ & $0.943 \pm 0.071$ & $0.922 \pm 0.078$ & $0.917 \pm 0.080$ & $<0.001$ \\
\hline $\mathrm{SBP}, \mathrm{mmHg}$ & $120.7 \pm 18.7$ & $125.9 \pm 20.2$ & $120.3 \pm 18.5$ & $118.1 \pm 17.3$ & $<0.001$ \\
\hline $\mathrm{DBP}, \mathrm{mmHg}$ & $73.3 \pm 11.1$ & $74.8 \pm 12.2$ & $72.8 \pm 10.8$ & $72.9 \pm 10.7$ & $<0.001$ \\
\hline Hypertension & 3,837 (31.7) & $1,174(44.1)$ & 1,499 (29.9) & $1,164(26.2)$ & $<0.001$ \\
\hline DM & $1,032(8.5)$ & $384(14.4)$ & $388(7.7)$ & $260(5.9)$ & $<0.001$ \\
\hline CAD & $503(4.2)$ & $176(6.6)$ & $189(3.8)$ & $138(3.1)$ & $<0.001$ \\
\hline Smoking & & & & & $<0.001$ \\
\hline Current smoker & 3,127 (25.8) & $898(33.7)$ & 1,348 (26.9) & $881(19.9)$ & \\
\hline Former smoker & 3,985 (32.9) & $814(30.6)$ & 1,569 (31.3) & $1,602(36.1)$ & \\
\hline Never smoker & $4,997(41.3)$ & $952(35.7)$ & $2,090(41.7)$ & $1,955(44.1)$ & \\
\hline Drinking & & & & & $<0.001$ \\
\hline Current drinker & 7,053 (58.2) & 995 (37.3) & $2,959(59.1)$ & $3,099(69.8)$ & \\
\hline Former drinker & $2,225(18.4)$ & $811(30.4)$ & 864 (17.3) & $550(12.4)$ & \\
\hline Never drinker & $2,831(23.4)$ & $858(32.2)$ & $1,184(23.6)$ & $789(17.8)$ & \\
\hline Income, US\$/year & & & & & $<0.001$ \\
\hline$\leq 34,999$ & $6,488(53.6)$ & $2,280(85.6)$ & $2,824(56.4)$ & $1,384(31.2)$ & \\
\hline$\geq 35,000$ & $5,621(46.4)$ & $384(14.4)$ & $2,183(43.6)$ & $3,054(68.8)$ & \\
\hline Sports & $2.5 \pm 0.8$ & $2.2 \pm 0.7$ & $2.4 \pm 0.8$ & $2.6 \pm 0.8$ & $<0.001$ \\
\hline Physical activity & $2.4 \pm 0.6$ & $2.1 \pm 0.6$ & $2.4 \pm 0.6$ & $2.5 \pm 0.5$ & $<0.001$ \\
\hline $\mathrm{TC}, \mathrm{mmol} / \mathrm{L}$ & $5.6 \pm 1.1$ & $5.7 \pm 1.1$ & $5.6 \pm 1.0$ & $5.4 \pm 1.0$ & $<0.001$ \\
\hline $\mathrm{HDL}-\mathrm{C}, \mathrm{mmol} / \mathrm{L}$ & $1.3 \pm 0.4$ & $1.3 \pm 0.4$ & $1.3 \pm 0.4$ & $1.4 \pm 0.4$ & 0.003 \\
\hline LDL-C, mmol/L & $3.6 \pm 1.0$ & $3.7 \pm 1.0$ & $3.6 \pm 1.0$ & $3.5 \pm 1.0$ & $<0.001$ \\
\hline $\mathrm{TG}, \mathrm{mmol} / \mathrm{L}$ & $1.4 \pm 0.7$ & $1.4 \pm 0.7$ & $1.4 \pm 0.7$ & $1.4 \pm 0.7$ & $<0.001$ \\
\hline $\mathrm{Cr}, \mathrm{mg} / \mathrm{dL}$ & $1.1 \pm 0.4$ & $1.1 \pm 0.5$ & $1.1 \pm 0.4$ & $1.1 \pm 0.3$ & $<0.001$ \\
\hline UN, mg/dL & $15.2 \pm 4.1$ & $15.5 \pm 5.0$ & $15.2 \pm 3.9$ & $15.2 \pm 3.8$ & 0.200 \\
\hline HTN medication & $3,243(26.8)$ & $965(36.2)$ & $1,279(25.5)$ & $999(22.5)$ & $<0.001$ \\
\hline Statin use & $65(0.5)$ & $15(0.6)$ & $29(0.6)$ & $21(0.5)$ & 0.764 \\
\hline Aspirin use & $5,695(47.0)$ & $1,131(42.5)$ & $2,473(49.4)$ & $2,347(47.1)$ & $<0.001$ \\
\hline
\end{tabular}

Data are presented as mean $\pm \mathrm{SD}$ or number (percentage). BMI, body mass index; $\mathrm{CAD}$, coronary artery disease; $\mathrm{Cr}$, creatinine; DBP, diastolic blood pressure; DM, diabetes mellitus; HDL-C, high-density lipoprotein cholesterol; HTN, hypertension LDL-C, low-density lipoprotein cholesterol; SBP, systolic blood pressure; TC, total cholesterol; TG, triglycerides; UN, urea nitrogen; WHR, waist-to hip ratio.

measured by trained nurses. BMI and WHR were calculated as the ratio of weight $(\mathrm{kg})$ to the square of height $\left(\mathrm{m}^{2}\right)$, and the ratio of waist $(\mathrm{cm})$ to hip circumference $(\mathrm{cm})$, respectively.

\section{Covariates}

All covariates included in our regression model were evaluated at the ARIC baseline visit using standardized methods. ${ }^{12}$ Demographic variables including age, race and sex were self-reported. We included the following laboratory indexes as covariates: creatinine $(\mathrm{Cr})$, urea nitrogen (UN), total cholesterol (TC), high-density lipoprotein cholesterol (HDL-C), low-density lipoprotein cholesterol (LDL-C) and triglycerides (TG). Hypertension (HTN) was identified if a participant had systolic blood pressure (SBP) $\geq 140 \mathrm{mmHg}$ or diastolic blood pressure (DBP) $\geq 90 \mathrm{mmHg}$, antihypertensive drug use, or prior history of hypertension. We defined diabetes mellitus (DM) as fasting glucose $\geq 126 \mathrm{mg} / \mathrm{dL}$, non-fasting glucose of $\geq 200 \mathrm{mg} / \mathrm{dL}$, self-reported history of
DM or use of DM medications. Prevalent coronary artery disease (CAD) was defined as self-reported history of myocardial infarction (MI), coronary revascularization or MI from adjudicated visit 1 ECG data. The definition of prevalent stroke is described elsewhere. ${ }^{19}$ Medication use 2 weeks before the investigation was determined through self-report.

\section{Statistical Analysis}

Participants were divided into 3 groups based on educational attainment. baseline characteristics were then demonstrated based on one-way ANOVA (continuous variables), $\chi^{2}$ tests (categorical variables) and the Kruskal-Wallis test. The data are shown as mean \pm SD or frequency (percentage).

Kaplan-Meier curves were drawn to estimate the proportion of remaining survival free from HF for the 3 education group. Univariable Cox regression models were performed to identity covariates $(\mathrm{P}<0.15$, Supplementary Table 1$)$. We graphically examined the assumption of the Cox regression 


\begin{tabular}{|c|c|c|c|}
\hline Model & HR $(95 \% \mathrm{Cl})$ & $P$ value & HR $(95 \% \mathrm{Cl})$ \\
\hline \multicolumn{4}{|l|}{ Model $1^{a}$} \\
\hline Low educational attainment & $1.94(1.75-2.16)$ & $<0.001$ & \\
\hline Medium educational attainment & $1.29(1.17-1.43)$ & $<0.001$ & $\rightarrow-$ \\
\hline \multicolumn{4}{|l|}{ Model $2^{b}$} \\
\hline Low educational attainment & $1.47(1.32-1.64)$ & $<0.001$ & $\rightarrow-$ \\
\hline Medium educational attainment & $1.14(1.03-1.27)$ & 0.023 & $\rightarrow-$ \\
\hline \multicolumn{4}{|l|}{ Model $3^{c}$} \\
\hline Low educational attainment & $1.41(1.26-1.57)$ & $<0.001$ & $\longrightarrow$ \\
\hline Medium educational attainment & $1.13(1.02-1.25)$ & 0.023 & $\rightarrow$ \\
\hline
\end{tabular}

Figure 2. Association of educational attainment with incident heart failure. aModel 1: adjusted for age, race and sex. ${ }^{\mathrm{b}} \mathrm{Model} 2$ : further adjusted for BMI, WHR, smoking status, drinking status. 'Model 3: further adjusted for HTN, DM, CAD, stroke, TC, HDL-C, LDL-C, TG, UN, Cr, HTN MED, statin use and aspirin use. BMI, body mass index; CAD, coronary artery disease; Cl, confidence interval; Cr, creatinine; DM, diabetes mellitus; HR, hazard ratio; HDL-C, high-density lipoprotein cholesterol; HTN, hypertension; LDL-C, low-density lipoprotein cholesterol; TC, total cholesterol; TG, triglycerides; UN, urea nitrogen; WHR, waist-to-hip ratio.

model (Supplementary Figure 1). Multivariable Cox regression models with high educational attainment as the reference were performed to assess the association between educational attainment and incident HF. Model 1 was adjusted for age, race and sex. Model 2 was further adjusted for BMI, WHR, smoking, drinking. Model 3 was additionally adjusted for HTN, DM, CAD, stroke, TC, HDL-C, LDL-C, TG, UN, Cr, antihypertensive medication, statin and aspirin use. To investigate potential causal pathways linking educational attainment to $\mathrm{HF}$, we use the mediation model constructed by Baron and Kenny. ${ }^{20,21}$ The concept of mediation analysis is provided in Supplementary Methods and Figure 1. We investigated the association between potential mediators and educational attainment through linear regression (continuous variables) and logistic regression (categorical variables). The associations between potential mediators with incident HF were tested based on the Cox regression model. We calculated the direct and indirect effects of potential mediators by singly adding each mediator into the basic mediation model then withdrawing it for the next operation. Basic mediation models were adjusted for age, race and sex in COX regression model. The proportion of effect mediated (PM) by the mediator is defined as the indirect effect/total effect. To simply the mediation model, we assessed the effect between low and high educational attainment. We performed competing risk model accounting for competing risks of death in the sensitivity analysis. We also examined the association between educational attainment with $\mathrm{HF}$ and the mediating role of behavioral factors in individuals free from HTN, $\mathrm{DM}$ and CAD at baseline. In addition, subgroup analysis and trend tests were performed grouped by age, race, sex, income, current smoking, current drinking, BMI, WHR, sports and physical activity. Interaction between educational attainment and these covariates were tested.

Statistical analysis was performed using $\mathrm{R}$ version 3.5.3 and STATA 15.1. Mediation analysis was conducted using package "Mediation". ${ }^{21}$ A $\mathrm{P}$ value $<0.05$ was considered to be statistically significant.

\section{Results}

\section{Baseline Characteristics}

The baseline characteristics of 12,109 participants stratified by educational attainment are shown in Table 1 . The mean age of participants was 54.2 \pm 5.6 years, 5,621 (46.4\%) were male and 9,386 (77.5\%) were white. Individuals with low educational attainment were older, more frequently current smokers, had low income, and undertook less sports and physical activity. They also tended to have high values for BMI, WHR, SBP, DBP, TC, TG, LDL-C, Cr and high prevalence of HTN, stroke, DM, CAD, HTN medication and aspirin use.

Association Between Educational Attainment and Incident HF The Kaplan-Meier curves showed the highest cumulative probability of incident $\mathrm{HF}$ in individuals with low educational attainment $(\mathrm{P}<0.0001$ from log-rank test; Supplementary Figure 2). Figure 2 shows the results using Cox proportional hazard regression models. In Model 1 adjusted for sex, race and age, individuals with low and medium educational attainment had significantly increased HF risk compared with individuals with high educational attainment. In Model 2, we observed an attenuated but still significant effect after further adjusting for BMI, WHR, smoking and drinking. Additional adjustment for HTN, DM, CAD, stroke, TC, HDL-C, LDL-C, TG, UN, $\mathrm{Cr}, \mathrm{HTN}$ medication, statin and aspirin use in Model 3 did not change the association.

\section{Association Between Educational Attainment and Potential Mediators}

There were significant associations between educational attainment and all potential mediators (Supplementary Table 2). We found a gradient for the hazard ratio (HR) of all potential mediators across educational attainment 


\begin{tabular}{|c|c|c|c|c|c|}
\hline \multirow{3}{*}{ Model } & \multicolumn{5}{|c|}{ Mediation analysis } \\
\hline & Low & Medium & Higha $^{a}$ & RM O/b & D ulun \\
\hline & HR $(95 \% \mathrm{Cl})$ & HR $(95 \% \mathrm{Cl})$ & HR & PIVI, $70^{\circ}$ & Pralue \\
\hline Base $^{c}$ & $1.94(1.75-2.16)$ & $1.29(1.17-1.43)$ & 1.00 & - & - \\
\hline +Income $\geq U S \$ 35,000$ & $1.68(1.50-1.88)$ & $1.18(1.07-1.31)$ & 1.00 & 24.3 & $<0.001$ \\
\hline+ WHR & $1.74(1.56-1.93)$ & $1.25(1.13-1.38)$ & 1.00 & 20.2 & $<0.001$ \\
\hline+ Current smoking & $1.80(1.62-2.00)$ & $1.23(1.11-1.36)$ & 1.00 & 13.8 & $<0.001$ \\
\hline +BMI & $1.82(1.63-2.02)$ & $1.27(1.15-1.41)$ & 1.00 & 10.1 & $<0.001$ \\
\hline+ Current drinking & $1.85(1.66-2.06)$ & $1.27(1.15-1.40)$ & 1.00 & 7.7 & 0.014 \\
\hline+ Sports & $1.85(1.66-2.06)$ & $1.26(1.14-1.39)$ & 1.00 & 7.3 & $<0.001$ \\
\hline +Physical activity & $1.89(1.70-2.11)$ & $1.28(1.16-1.42)$ & 1.00 & 4.5 & $<0.001$ \\
\hline Total $^{d}$ & $1.34(1.19-1.50)$ & $1.08(0.97-1.19)$ & 1.00 & 56.3 & - \\
\hline
\end{tabular}

aReference category. ${ }^{b}$ Proportion mediated. ${ }^{~}$ Adjusting for age, race and sex. ${ }^{d}$ Adjusting for age, race, sex, BMI, WHR, smoking status, drinking status, income, sports and physical activity. Abbreviations as in Table 1.

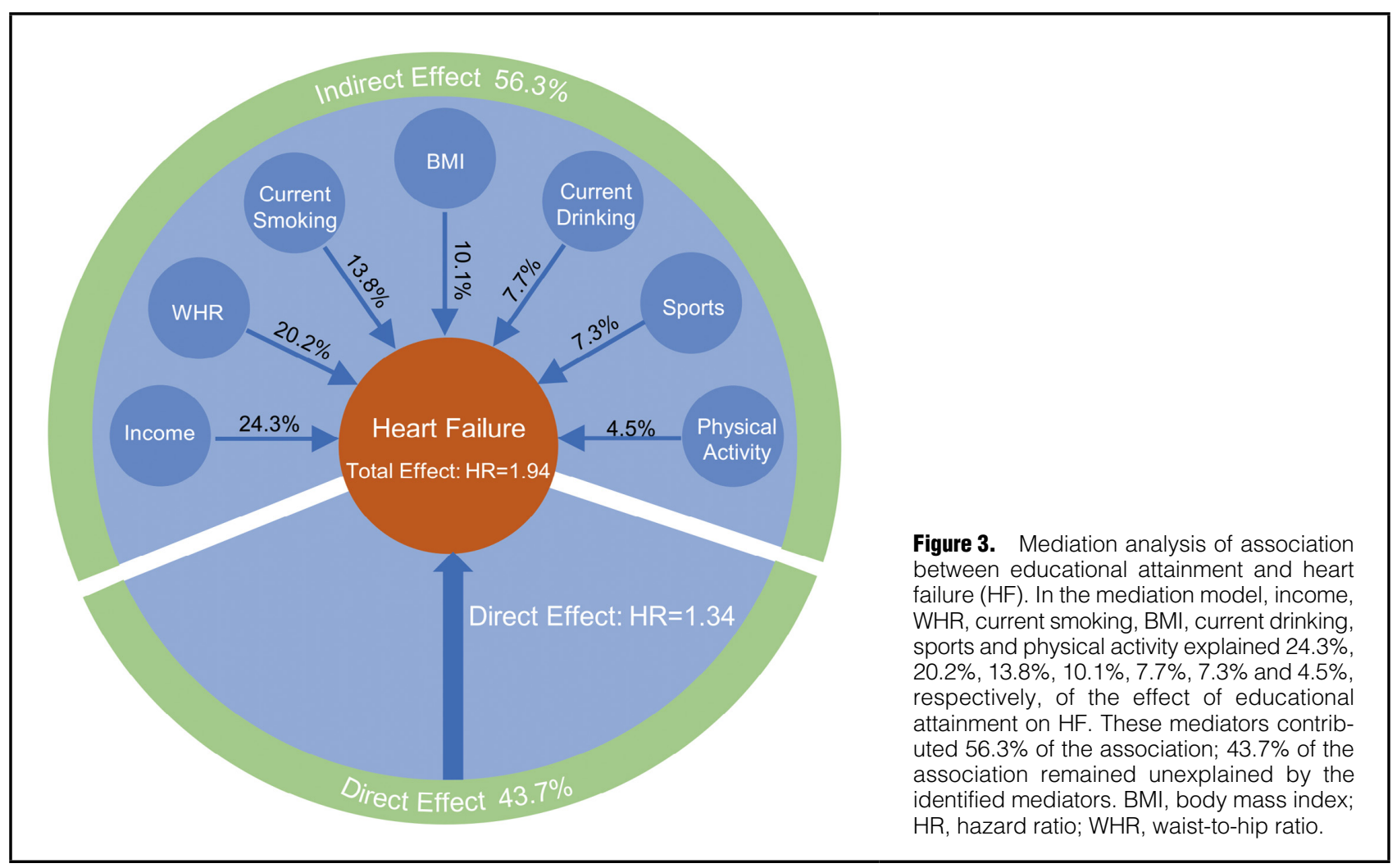

groups. When compared with high educational attainment, the prevalence of current smoking, BMI and WHR increased significantly with both medium and low educational attainment. Less-educated individuals were more likely to have low levels of sports, physical activity, income and a low prevalence of current drinking.

\section{Association Between Potential Mediators and Incident HF}

All potential mediators were significantly associated with HF (Supplementary Figure 3). Current smoking, BMI and WHR were associated with increased risks of incident $\mathrm{HF}$ in the Cox regression model adjusted for sex, race and age. Conversely, higher income ( $\geq$ US $\$ 35,000 /$ year), current drinking, sports and physical activity were associated with decrease hazard of incident HF.

\section{Mediation Analysis}

The results of mediation analysis are displayed in Table 2 and Figure 3. The association between educational attainment and HF was partly mediated by income, WHR, current smoking, BMI, current drinking, sports and physical activity, which explained $24.3 \%, 20.2 \%, 13.8 \%, 10.1 \%, 7.7 \%$, $7.3 \%$ and $4.5 \%$, respectively, of the total effect. Overall, the included mediators explained $56.3 \%$ of the association.

\section{Sensitivity Analysis}

An inverse association between educational attainment and $\mathrm{HF}$ was also observed in the competing risk model 
(Supplementary Table 3). Analyses limited to those free from CAD, HTN and DM history $(n=1,446)$ showed similar findings (Supplementary Table 4A,B). Income, obesity and smoking remained the most important mediators, and drinking did not achieve statistical significance. In the subgroup analysis, the associations between educational attainment and incident HF were stronger in younger, white, current smoker, former or never drinker, individuals with BMI $\geq 25.0$ or WHR $\geq 0.93$ (Supplementary Tables 5-14). Interactions were statistically significant between age and educational attainment, WHR and educational attainment ( $\mathrm{P}$ for interaction $<0.05$, Supplementary Tables 5,12).

\section{Discussion}

In our analysis of 12,109 participants from the ARIC study, we found that educational attainment was inversely associated with the risk of incident HF, which increased progressively from well-educated participants to those less educated. Based on mediation analysis, we identified several behavioral mediators involving in the association of low educational attainment in incident $\mathrm{HF}$, which contributed to $56.3 \%$ of the effect and mostly through income, WHR, current smoking and BMI (Table 2). These findings revealed a potential causal pathway of the relationship, thus providing a basis for new interventional strategies to ameliorate the educational disparities in HF occurrence. In addition, several easily measured mediators in clinical work make it possible to identify less-educated individuals at high risk.

Although some existing studies have assessed the association between educational attainment and HF, 3,4,22 to our knowledge, this is the first to investigate the potential mechanisms underlying the relationship using mediation analysis. In the NHANES, which followed 13,643 participants for 20 years, less-educated participants had a relative risk of incident HF of 1.22 (95\% confidence interval (CI), 1.04-1.42). ${ }^{22}$ Likewise, in a prospective study involving with 2,314 middle-aged men, Ingelsson and colleagues reported the HR for HF was 1.98 (95\% CI, 1.07-3.68) for less-educated individuals after multivariable adjustment. ${ }^{4}$ It should be noted that a detrimental effect of low educational attainment on CVD has been reported in the ARIC study, concerning a composite outcome of CAD, HF and stroke. ${ }^{23}$ Less-educated subjects tended to have higher levels of CVD risk factors in that study, such as more obesity, more frequently smoking and so on. Income levels varied across educational groups and contributed to the divergent clinical outcomes. However, whether or not these behavioral factors mediate the deleterious effects of low educational attainment was still unclear. Our results were in accord with those from previous studies, so we further conducted a mediation analysis to explore the underlying mechanism.

\section{Interpretation and Public Health Implications}

To radically reduce the educational disparities in HF incidence, interventions for enhancing adolescents' educational attainment might be effective. But for those who have finished school, whether they can benefit from further education is unclear. Indeed, education is usually completed in early life and tends to be stable throughout one's life course such that lower educational attainment might expose individuals to HF risk factors from young age. Even if educational attainment is improved later, the cumulative risk of HF might not be reversed easily. Tarani et al found adult education was associated with a slight reduction in the risk of CAD. ${ }^{24}$ Therefore, further study is required to estimate whether additional education can reduce the risk of HF in less-educated individuals.

Income, which explained $24.3 \%$ of the total effect, was the strongest mediator among all those examined in our study. In our research, lower educational attainment was associated with low income level. In fact, individuals with low income might have poor affordability, less access to healthcare facilities and less social support. To be specific, low-income individuals might not be able to afford a healthier diet, which may be relatively more expensive, such as fruits. ${ }^{\mathbf{2 5 , 2 6}}$ Besides, individuals with lower income may reside in either inner-city or rural areas with inconvenient access to healthcare facilities. ${ }^{25}$ In addition, they may also have less social support due to lack of social resources. Thus, policies targeting individuals with lower income (e.g., increasing the minimum wage) might be beneficial for improving the educational inequalities in $\mathrm{HF}$.

Our findings showed that BMI and WHR mediated a considerable portion of the relationship, but WHR mediated more effect than BMI (20.2\% vs. 10.1\%). Less-educated individuals were more likely to have higher BMI and WHR, thus leading to increased HF risk. This is also supported by observations from prior studies. ${ }^{27,28}$ General obesity, measured by BMI, is a well-recognized risk factor for CVD, including HF. ${ }^{27,29}$ Although BMI is extensively used to assess obesity, it seems unable to depict abdominal adiposity accurately, thus not capturing the corresponding deleterious effect. Notably, Russo et al reported that higher WHR correlated with subclinical LV systolic dysfunction across BMI categories, ${ }^{30}$ whereas abdominal obesity measured by waist circumference was associated with incident $\mathrm{HF}$ even after adjustment for BMI. ${ }^{31}$ These observations suggest that abdominal obesity may exert an deleterious effect on HF independent of general obesity; however, no direct evidence was obtained to support the effect of WHR on incident HF accounting for BMI, which needs future research to clarify. As an exploratory analysis, we used BMI and WHR to represent 2 types of obesity and revealed a more predominant role of abdominal obesity in mediating the effect of education on HF. Considering that weight loss is recommended as an effective preventive strategy for $\mathrm{HF},{ }^{32}$ comprehensive lifestyle interventions targeting weight loss, such as increasing sports involvement and calorie restriction, should be recommended to improve the risk of $\mathrm{HF}$ in less-educated individuals. Meanwhile, given the significant mediating effects of WHR in less-educated subjects, a strategy towards prevention and management of abdominal obesity may reduce the burden of $\mathrm{HF}$.

Current smoking, a lifestyle-related factor, played an important role in the causal pathway. ${ }^{13}$ Similar to previous findings, educational attainment was inversely associated with the prevalence of current smoking. ${ }^{33}$ The mediating role of current smoking can be interpreted asa causal effect of lack of health literacy and self-control in less-educated populations, ${ }^{34,35}$ suggesting that interventions targeting smoking cessation, such as smoking cessation counseling and pre-cessation medication use, should be carried out to block the casual pathway.

The pathogenesis of HF is complex and multifactorial, and seems to be a cascade pathway. The behavioral factors serve as upstream mediators in the casual pathway between educational attainment and HF. Behavioral disparities 
derived from educational attainment may have subsequent effects on other diseases such as CAD and DM, known as risk factors of HF. ${ }^{\mathbf{7} 36}$ In a word, educational inequality affected the incidence of some diseases through behavioral factors, ultimately interfering in the onset of HF. As a first step, identification of behavioral mediators will be helpful for breaking the deleterious effect of educational inequality.

A noteworthy finding in the sensitivity analysis was that individuals less than 55 years old were vulnerable to the educational inequality effect on HF risk. A possible explanation is that the effect of educational attainment varies over time. Older participants benefited less from high educational attainment due to low living standards in the past. For younger participants, with the improvements in living standards, the effects of educational inequality were amplified and more benefits from high educational attainment occurred. Therefore, low educational attainment in the young should be heeded for its increased HF risk.

For policymakers, we hope the findings of our analysis can help to design public health interventions targeting identified mediators. Now that income status, obesity and smoking are identified as important mediators, interventions targeting them might become the cornerstone of policies developed to improve educational inequalities in $\mathrm{HF}$ risk. For researchers, $43.7 \%$ of the total effect was attributed to direct effect in the mediation analysis model and to be exact, it was the part of the effect not accounted for by the selected mediators. So, some unmeasured mediators need to be investigated in future, such as psychosocial stress, blood lipid profile. What's more, to our knowledge, there are no established guidelines or risk scoring of HF concerning educational attainment and the corresponding mediators, ${ }^{32}$ which deserves extra attention in future research.

\section{Study Limitations}

There are several limitations in our study to be considered. First, we evaluated the risk of educational attainment on HF using information obtained at baseline. However, there might be some individuals who underwent further education after visit 1 , which could lead to bias. Second, we had no information on the age of individuals when they completed their highest educational attainment. Different ages of completing education might result in discrepancy of exposure to risk factors. Third, categorizing the educational level into 3 groups may be rough to some extent due to the mixture of effects derived of educational disparities in the same group, finally leading to loss of information in each educational group. Fourth, incident HF was ascertained through hospital discharge codes. Hence, individuals who were managed in the outpatient department would be ignored. Meanwhile, we did not consider 2 distinct types of HF: HF with reduced ejection fraction and preserved ejection fraction. Fifth, the results were mainly derived from the white population (77.5\%) in America. Indeed, educational attainment and behavioral factors may vary among different regions and ethnicities, which could possibly bias the conclusions. Hence, extrapolation of the findings to other populations should be undertaken with caution and additional validation is required to verify these results. Sixth, to highlight current behavioral health risks, we only used "current drinking" and "current smoking" as potential mediators. Our study did not address whether former drinking and former smoking mediate the effect of educational inequality on HF risk, which warrants clarification in future research. Finally, due to the nature of the obser- vational design, residual confounding could not be eliminated despite adjustment of covariates as much as possible, precluding assessment of causation.

\section{Conclusions}

In conclusion, educational attainment was inversely associated with incident $\mathrm{HF}$, mediated mainly by income, obesity and smoking. This overall understanding opens a new chapter for designing targeted public health interventions based on the potential causal pathway between educational attainment and $\mathrm{HF}$.

\section{Acknowledgments}

We thank the staff and participants of the ARIC study for their contributions.

\section{Author Contributions}

X.L., X. Zhuang and LYF contributed to the conception or design of the work. All authors were responsible for the acquisition, analysis and interpretation of data. Y. Lin, S.Z. and S.W. drafted the manuscript. Critical revision of the manuscript for important intellectual content was performed by all authors. All author agreed with the content of the article to be submitted.

\section{IRB Information}

This study is a secondary data analysis of de-identified data from the ARIC study (https://www2.cscc.unc.edu/aric/). The ARIC study protocol was approved by the institutional review board of each participating university. Therefore, the Ethics Committee of the First Affiliated Hospital of Sun Yat-Sen University decided this study did not need ethics approval.

\section{Funding}

This study was supported by the National Natural Science Foundation of China (81600206 to X. Zhuang; 81870195 to X.L.), and Natural Science Foundation of Guangdong Province (2016A030310140 to X. Zhuang; 20160903 to X.L.).

\section{Declaration of Interests}

The authors have no conflicts of interest to disclose.

\section{References}

1. Benjamin EJ, Muntner P, Alonso A, Bittencourt MS, Callaway CW, Carson AP, et al. Heart disease and stroke statistics-2019 update: A report from the American Heart Association. Circulation 2019; 139: e 56-e 528.

2. Echouffo-Tcheugui JB, Bishu KG, Fonarow GC, Egede LE. Trends in health care expenditure among US adults with heart failure: The Medical Expenditure Panel Survey 2002-2011. Am Heart J 2017; 186: $63-72$.

3. Christensen S, Mogelvang R, Heitmann M, Prescott E. Level of education and risk of heart failure: A prospective cohort study with echocardiography evaluation. Eur Heart $J$ 2011; 32: 450458.

4. Ingelsson E, Lind L, Arnlov J, Sundstrom J. Socioeconomic factors as predictors of incident heart failure. $J$ Card Fail 2006; 12: $540-545$.

5. Di Chiara T, Scaglione A, Corrao S, Argano C, Pinto A, Scaglione $\mathrm{R}$. Association between low education and higher global cardiovascular risk. J Clin Hypertens (Greenwich) 2015; 17: 332-337.

6. Reddy KS, Prabhakaran D, Jeemon P, Thankappan KR, Joshi $\mathrm{P}$, Chaturvedi V, et al. Educational status and cardiovascular risk profile in Indians. Proc Natl Acad Sci USA 2007; 104: $16263-$ 16268.

7. Nordahl H, Rod NH, Frederiksen BL, Andersen I, Lange T, Diderichsen $\mathrm{F}$, et al. Education and risk of coronary heart disease: Assessment of mediation by behavioral risk factors using the additive hazards model. Eur J Epidemiol 2013; 28: 149-157.

8. Schultz WM, Kelli HM, Lisko JC, Varghese T, Shen J, Sandesara $\mathrm{P}$, et al. Socioeconomic status and cardiovascular outcomes: 
Challenges and interventions. Circulation 2018; 137: 2166-2178.

9. Albert MA, Glynn RJ, Buring J, Ridker PM. Impact of traditional and novel risk factors on the relationship between socioeconomic status and incident cardiovascular events. Circulation 2006; 114: 2619-2626.

10. Beauchamp A, Peeters A, Wolfe R, Turrell G, Harriss LR, Giles $\mathrm{GG}$, et al. Inequalities in cardiovascular disease mortality: The role of behavioural, physiological and social risk factors. $J$ Epidemiol Community Health 2010; 64: 542-548.

11. Kivimaki M, Lawlor DA, Davey Smith G, Kouvonen A, Virtanen M, Elovainio M, et al. Socioeconomic position, co-occurrence of behavior-related risk factors, and coronary heart disease: The Finnish Public Sector study. Am J Public Health 2007; 97: $874-879$.

12. Investigators TA. The Atherosclerosis Risk in Communities (ARIC) Study: Design and objectives. The ARIC investigators. Am J Epidemiol 1989; 129: 687-702.

13. Kamimura D, Cain LR, Mentz RJ, White WB, Blaha MJ, DeFilippis AP, et al. Cigarette smoking and incident heart failure: Insights from the Jackson Heart Study. Circulation 2018; 137: $2572-2582$

14. Ng R, Sutradhar R, Yao Z, Wodchis WP, Rosella LC. Smoking, drinking, diet and physical activity-modifiable lifestyle risk factors and their associations with age to first chronic disease. Int $J$ Epidemiol 2020; 49: 113-120.

15. Potter EL, Hopper I, Sen J, Salim A, Marwick TH. Impact of socioeconomic status on incident heart failure and left ventricular dysfunction: Systematic review and meta-analysis. Eur Heart J Qual Care Clin Outcomes 2019; 5: 169-179.

16. Aune D, Sen A, Norat T, Janszky I, Romundstad P, Tonstad S, et al. Body mass index, abdominal fatness, and heart failure incidence and mortality: A systematic review and dose-response meta-analysis of prospective studies. Circulation 2016; 133: 639-649.

17. Jacobs DR Jr, Ainsworth BE, Hartman TJ, Leon AS. A simultaneous evaluation of 10 commonly used physical activity questionnaires. Med Sci Sports Exerc 1993; 25: 81 -91.

18. Hertogh EM, Monninkhof EM, Schouten EG, Peeters PH, Schuit AJ. Validity of the modified Baecke questionnaire: Comparison with energy expenditure according to the doubly labeled water method. Int J Behav Nutr Phys Act 2008; 5: 30.

19. Folsom AR, Nambi V, Bell EJ, Oluleye OW, Gottesman RF, Lutsey PL, et al. Troponin T, N-terminal pro-B-type natriuretic peptide, and incidence of stroke: The Atherosclerosis Risk in Communities study. Stroke 2013; 44: 961-967.

20. Baron RM, Kenny DA. The moderator-mediator variable distinction in social psychological research: Conceptual, strategic, and statistical considerations. J Pers Soc Psychol 1986; 51: 1173 1182 .

21. Tingley D, Yamamoto T, Hirose K, Keele L, Imai K. Mediation: R Package for causal mediation analysis. J Stat Softw 2014; 5: $1-38$.

22. He J, Ogden LG, Bazzano LA, Vupputuri S, Loria C, Whelton PK. Risk factors for congestive heart failure in US men and women: NHANES I epidemiologic follow-up study. Arch Intern Med 2001; 161: 996-1002.

23. Kubota Y, Heiss G, MacLehose RF, Roetker NS, Folsom AR. Association of educational attainment with lifetime risk of cardiovascular disease: The Atherosclerosis Risk in Communities
Study. JAMA Intern Med 2017; 177: 1165-1172.

24. Chandola T, Plewis I, Morris JM, Mishra G, Blane D. Is adult education associated with reduced coronary heart disease risk? Int J Epidemiol 2011; 40: 1499-1509.

25. Rosengren A, Smyth A, Rangarajan S, Ramasundarahettige C, Bangdiwala SI, AlHabib KF, et al. Socioeconomic status and risk of cardiovascular disease in 20 low-income, middle-income, and high-income countries: The Prospective Urban Rural Epidemiologic (PURE) study. Lancet Glob Health 2019; 7: e748-e760.

26. Rautiainen S, Levitan EB, Mittleman MA, Wolk A. Fruit and vegetable intake and rate of heart failure: A population-based prospective cohort of women. Eur J Heart Fail 2015; 17: 20-26.

27. Kenchaiah S, Evans JC, Levy D, Wilson PW, Benjamin EJ, Larson MG, et al. Obesity and the risk of heart failure. $N$ Engl $J$ Med 2002; 347: 305-313.

28. Hales CM, Carroll MD, Fryar CD, Ogden CL. Prevalence of obesity among adults and youth: United States, 2015-2016. NCHS Data Brief 2017; (288): 1-8.

29. Hubert HB, Feinleib M, McNamara PM, Castelli WP. Obesity as an independent risk factor for cardiovascular disease: A 26-year follow-up of participants in the Framingham Heart Study. Circulation 1983; 67: 968-977.

30. Russo C, Sera F, Jin Z, Palmieri V, Homma S, Rundek T, et al. Abdominal adiposity, general obesity, and subclinical systolic dysfunction in the elderly: A population-based cohort study. Eur J Heart Fail 2016; 18: 537-544.

31. Nicklas BJ, Cesari M, Penninx BW, Kritchevsky SB, Ding J, Newman A, et al. Abdominal obesity is an independent risk factor for chronic heart failure in older people. J Am Geriatr Soc 2006; 54: 413-420.

32. Ponikowski P, Voors AA, Anker SD, Bueno H, Cleland JGF, Coats AJS, et al. 2016 ESC guidelines for the diagnosis and treatment of acute and chronic heart failure: The Task Force for the Diagnosis and Treatment of Acute and Chronic Heart Failure of the European Society of Cardiology (ESC); Developed with the special contribution of the Heart Failure Association (HFA) of the ESC. Eur Heart J 2016; 37: 2129-2200.

33. Steele CJ, Schottker B, Marshall AH, Kouvonen A, O'Doherty MG, Mons U, et al. Education achievement and type 2 diabetes: What mediates the relationship in older adults? Data from the ESTHER study: A population-based cohort study. BMJ Open 2017; 7: e013569.

34. Wilhelmsen L, Rosengren A, Eriksson H, Lappas G. Heart failure in the general population of men: Morbidity, risk factors and prognosis. J Intern Med 2001; 249: 253-261.

35. Serrano-Alarcon M, Kunst AE, Bosdriesz JR, Perelman J. Tobacco control policies and smoking among older adults: A longitudinal analysis of 10 European countries. Addiction 2019; 114: $1076-1085$.

36. Singh-Manoux A, Fayosse A, Sabia S, Tabak A, Shipley M, Dugravot A, et al. Clinical, socioeconomic, and behavioural factors at age 50 years and risk of cardiometabolic multimorbidity and mortality: A cohort study. PLoS Med 2018; 15: e1002571.

\section{Supplementary Files}

Please find supplementary file(s);

http://dx.doi.org/10.1253/circj.CJ-21-0109 